\title{
Remote Experts Help in Case of Emergency: A Cloud Computing Solution for Heart Disease Patients
}

\author{
Kavita S. Patil \\ Dept. of Electronics \&Telecommunication \\ Smt. Kashibai Navale College of Engineering \\ Pune, INDIA
}

\author{
Rohita H. Jagdale \\ Dept. of Electronics \& Telecommunication \\ Smt. Kashibai Navale College of Engineering \\ Pune, INDIA
}

\begin{abstract}
Time constraint is one of the important issues for the patients suffering from heart diseases. Delay in getting treatment can be life threatening. Essential data collection requires a great deal of efforts and time to collect and analyze the information. Such processes are usually slow and may become error-prone if done in a hurry. This situation limits the clinical diagnostics and monitoring capabilities. This paper presents the development of a remote monitoring system for ECG signals. Proposed solution is based on the concept of cloud computing and wireless networks. Analog signal is acquired with the ADC unit of the PIC24 controller, this information in terms of ECG is transferred through ZigBee network connected to the cloud and finally examination and storing are carried out. The signals obtained from the patients can be monitored simultaneously by the experts. Cloud provides availability, reliability.
\end{abstract}

\section{Keywords- ECG, Remote ECG capturing, Telemedicine}

\section{INTRODUCTION}

Coronary heart disorders are at the first place among the death causing diseases all over the world [1]. Health care peoples are trying to simplify the lives of the people suffering from chronic disorders like heart disease by using cost-effective and smart systems [2]. Heart disease is a common cause of death throughout the world and often it is not diagnosed early enough [3]. Electrocardiogram (ECG) signals are used to observe the electrical activity of the heart. It can detect abnormal heart rhythms, insufficient blood and oxygen supply to the heart and an excessive thickening of heart muscle. In recent years the emergency medical system has made great progress $[4,5,6]$. While the patient is shifted from his place to hospital, if patient's data could be sent to the cardiologist during this crucial period, by examining ECG signals, he may keep emergency systems on alert to save the life of the patient. He may instruct the attendant doctor in cardiac van to give necessary treatment. And if required then he may concern to other experts may be from abroad. One of the advantages of the proposed system over others of its kind is that it is cloud based system; the cloud provides availability, reliability. This is a tele-monitoring application. In telemonitoring application information and communication technologies are used to establish a virtual association between patients and their caregivers to support timely delivery of health-related data [7]. Here a solution is proposed that enables ECG to be transmitted and interpreted. The proposed system is cost effective; it provides availability and reliability and fault tolerance. ECG is derived from the biopotentials of the cardiac cell activities. At least two electrodes are required to be placed apart to measure the biopotential difference as the ECG [8]. In fact, the locations of those electrodes are well defined in cardiology as the ECG lead configurations. When multiple leads of ECG are monitored, it is essential to connect all leads to multichannel ECG amplifier so that the timing and synchronization of the ECGs are easily maintained during data acquisition [9]. Therefore, it is still imperative to use wired electrodes to collect ECG and other physiological signals from the patient and send the signals to the portable computing unit [6]. This unit has the responsibility of digitizing the analog signals, preprocessing the data and sending the data wirelessly to the data center in a cloud. For the proposed system ZigBee (IEEE 802.15.4) has been greeted as an ideal choice because it provides low power consumption, cost effective and standardized wireless networking solutions [10].

ZigBee can operate at $868 \mathrm{MHz}, 915 \mathrm{MHz}$, and $2.4 \mathrm{GHz}$ which are part of the industrial, scientific, and medical (ISM) $\mathrm{RF}$ bands. The data is stored on data server. The client can run on the browsers on MS Windows, Mac OS, Linux, iOS, and Android operating systems. It is possible to access a data on machine running with a web browser. Next section provides detail discussion on electrocardiography.

\subsection{Electrocardiography}

The countless circulating action within the heart creates a current flow, which in turn creates an electrical field. This electrical field can be detected, at the body surface, using a differential voltage measurement system. The resulting measurement is called as the electrocardiogram (ECG). The ECG signal is typically in the range of $2 \mathrm{mv}$ [11].

The ECG is a graphic representation of electrical activity of the hearts, can be recognized, \& interpreted as to normal or abnormal function. The ECG is also used to monitor the hearts response to the treatments. The ECG in a single cardiac cycle is shown in fig. 1. It contains a $\mathrm{P}$ wave, a QRS complex $\&$ a $\mathrm{T}$ wave $\&$ intervals are recorded as voltage Vs time. A waveform originates \& ends at the baseline. The waveform shows complex, the waveforms together are called a complex. A straight, line is called a segment. A waveform connecting to a segment is known an interval. The ECG waveforms above the reference line are considered as positive deflections and below the reference line are negative deflections. The contraction of the atria, generates the $\mathrm{P}$ wave. The size of the $\mathrm{P}$ wave is normally low. 


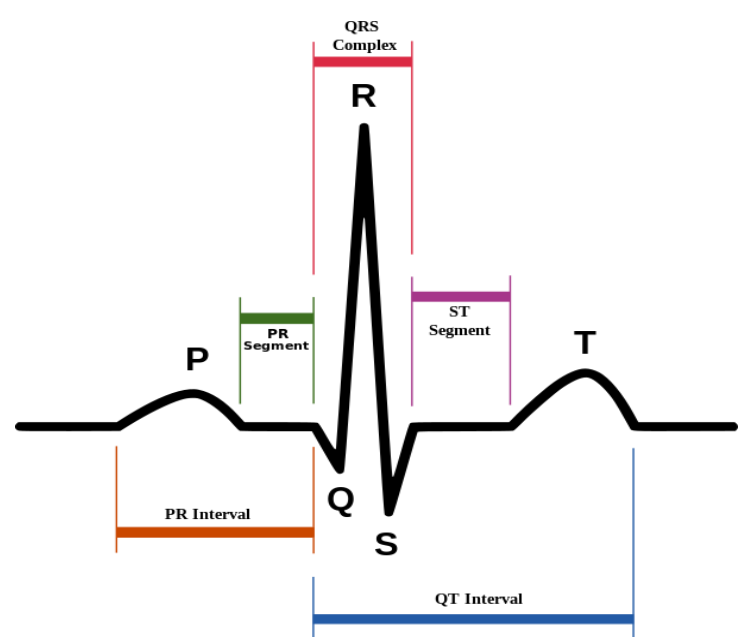

Fig. 1 ECG Diagram (From Wikipedia)

The PR interval begins with the start of the $\mathrm{P}$ wave \& ends at the start of $Q$ wave. It signifies the length of the conduction through the atria to the ventricals. It characterizes the length of conduction from the atrioventricular node, to the muscle. The QRS complex represents the time period of ventricular contraction. The QRS complex is much larger signal than the $\mathrm{P}$ wave due to the volume of ventricular tissue involved, If one of side of the heart is not working properly, the size of the QRS complex may increase.

The ST segment characterize the time period between the ventricular depolarisation $\&$ the repolarisation. The $\mathrm{T}$ wave is created due the repolarisation of the ventricles. It is having a longer time period as the ventricular repolarisation occurs slowly than depolarisation. The QT interval starts at Q wave $\&$ ends at the endpoint of the $\mathrm{T}$ wave representing the duration of the ventricular depolarisation repolarisation sequence [11].

\subsection{Cloud based systems}

In science, cloud computing is an implementation of distributed computing on a network and it is the ability to run a process on multiple networked computers at the same time. It is commonly used to denote to network based services which appear to be provided by real server hardware, which in fact are provide by virtual hardware, simulated with software running on one or more machines These virtual servers are not physically existent and can scale up.

Cloud computing depend on sharing of resources to obtain cost effectiveness for obtaining analogous to a utility a network. The cloud maximizes the utilization of the shared resources. Cloud resources are usually shared by multiple users as dynamically re-allocated per demand.

Cloud computing allows organizations to avoid infrastructure costs. Cloud computing allows organizations to get their applications always available, running faster, with less maintenance, and enables to fine-tune resources to meet varying and unpredictable business demand [12].

\section{RELATED WORK}

Various telemedicine systems are proposed by the researchers, some of the systems are discussed here. Hak Jong Leea et al. [8] proposed Ubiquitous healthcare service using Zigbee and mobile phone for elderly patients with objective to investigate the efficacy of a u-healthcare service using Zigbee and mobile phone for elderly patients with diabetes mellitus or heart diseases. 29 patients were enrolled in this study. Two parameters, ECG and blood glucose measurement were monitored. Twenty patients were using ZigBee incorporated blood glucometer and mobile phones. Using a web services the measured blood glucose could be transmitted and be administrated. Around nine patients participated in ECG monitoring, by using a wireless, ECG recording instrument equipped with ZigBee protocol attached on their chest. Every day's average transmission frequency, rate of transmission loss, and error causes were noted and analyzed. The patients were also asked to score their degree of satisfaction about the sensors and $\mathrm{u}$-healthcare services.

Wei Lin developed a real time monitoring of Electrocardiogram [1], it is still imperative to use wired electrodes to collect ECG and other physiological signals from the patient and send the signals to the portable computing unit. The proposed wireless platform adopts the star topology for the network configuration. Each patient is considered as one wireless node in the system and communicates with the access point directly using IEEE 802.15.4 protocol. The access point forwards the data to the data center over the Ethernet network. This configuration minimizes the number of wireless nodes and maximized the bandwidth for each patient for the real time streaming of physiological signals such as ECG.

Kyungtae Kang et al. [13] developed a real-time traffic information-based emergency medical service (RTIEMS) system by employing sensing devices, webcamera, ISM band RF module, GPS, Google Maps, ZigBee, and mobile network. In the ambulance, patient biosignals consisting of electrocardiogram (ECG), temperature, oxygen, and pulse can be wirelessly transmitted to the in-car gateway. Then, together with patient live video, these biosignals are transferred via WiMAX mobile network to the server located in the hospital emergency room for immediate preparation. In addition to it, to avoid traffic jam, a traffic guiding subsystem is provided based on the WebGIS that consists of Google Maps and GPS to help patient be transferred hospital with smallest time. Experimental results present the effectiveness of the proposed system in lengthening the golden rescue time.

S. Khoor et al. [14] added Bluetooth communication technology to their previously developed internet based information system, which collects the short and long term digitized ECGs with the relevant clinical data for the management of the patients. A wireless communication protocol was developed using the system for a short distance radio frequency data transmission.

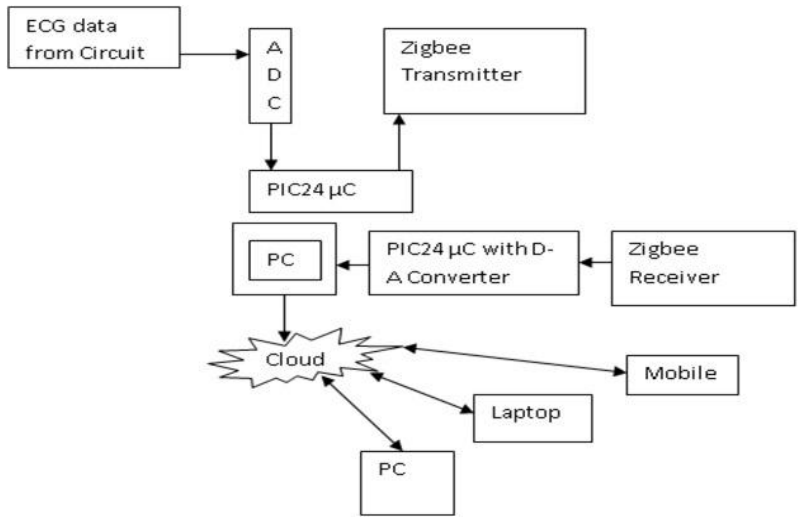

Fig. 2 Diagram Presenting The Proposed System 
Table 1. ZigBee Pro technology Specifications

\begin{tabular}{|l|l|}
\hline Specification & ZigBee pro \\
\hline Performance & Up to 300ft.(100m) \\
\hline Indoor/ Urban range & Up to 1mile(1500m) \\
\hline $\begin{array}{l}\text { Outdoor RF line-of- } \\
\text { sight range }\end{array}$ & $60 \mathrm{~mW}$ \\
\hline Transmit power output & $250,000 \mathrm{bps}$ \\
\hline RF data rate & $-100 \mathrm{dbm}$ \\
\hline Receiver sensitivity & $2.8 \mathrm{~V}-3.4 \mathrm{~V}$ \\
\hline Power requirement & $270 \mathrm{~mA}(@ 3.3 \mathrm{~V})$ \\
\hline Supply voltage & $55 \mathrm{~mA}(@ 3.3 \mathrm{~V})$ \\
\hline Transmit current & $<10 \mu \mathrm{A}$ \\
\hline Receive current & ISM $2.4 \mathrm{GHz}$ \\
\hline Power-down current & $0.960 \times 1.297$ \\
\hline General & $-40^{0}$ to $85^{\circ} \mathrm{C}$ \\
\hline Operating frequency \\
\hline Dimensions and \\
\hline Operating temperature \\
\hline $\begin{array}{l}\text { Networking } \\
\text { security }\end{array}$ \\
\hline $\begin{array}{l}\text { Supported Network } \\
\text { topologies }\end{array}$ & Point to point, Point to multipoint \\
\hline No. of channels & 13 direct sequence channels \\
\hline
\end{tabular}

\section{SYSTEM HARDWARE AND IMPLEMENTATION WORK}

The implementation details are discussed in next subsections.

\subsection{ECG Acquisition System}

The electrocardiogram (ECG) has become the most commonly conducted cardiovascular diagnostic procedure and a fundamental tool of clinical practice. Its interpretation may lead to the recognition of electrolyte abnormalities, mostly of serum potassium and calcium, and it permit the recognition of some forms of genetically mediated electrical or structural cardiac abnormalities. ECG signal is very weak low-frequency signal, amplitude 0.5 to $4 \mathrm{mV}$, frequency 0.05 to $100 \mathrm{~Hz}$, in the testing process mixed with other biological signals, $50 \mathrm{~Hz}$ frequency and the surrounding interference caused by electrical equipment. The ECG acquisition system consists of Electrodes, ECG supply circuit, Instrumentation amplifier, Chopper ckt, Filter, Preamplifier. AD620 IC is used for instrumentation amplifier. AD620 is low power, high accuracy instrumentation amplifier which requires only one external resistor to set gains. It is monolithic instrumentation amplifier based on a modification of three Op-amp approaches. The AD620 with its high accuracy of $40 \mathrm{ppm}$ maximum nonlinearity, lower offset voltage of $50 \mu \mathrm{V} \max$ with offset drift of $0.6 \mu \mathrm{V} /{ }^{0} \mathrm{C}$ max. The low current noise of AD620 allows its use in ECG monitors. A chopper circuit is used to refer to numerous types of electronic switching devices and circuits. Some types of signals that need amplifying can be so small that an incredibly high gain is needed, but high gain DC amplifiers are much harder to build with low offset and 1/f noise, and reasonable stability and reasonable bandwidth. It is far easier to prepare an AC amplifier in its place. A chopper circuit aims to break up the input signal so that it can be processed as if it were an $\mathrm{AC}$ signal, at that juncture it is integrated to a DC signal at the output. Thus, extremely minor DC signals can be amplified. Such approach is usually used in electronic instrumentation where stability and accuracy are essential. A high-pass filter (HPF) is an electronic filter that passes high-frequency signals and attenuates (reduces the amplitude of) signals with frequencies lower than the cutoff frequency. The real amount of attenuation for each frequency varies from filter to filter. A low pass filter is an electronic filter that passes low frequency signals and attenuates (reduces the amplitude of) signals with frequencies higher than the low limit of frequency. The authentic amount of attenuation for every frequency varies from filter to filter. It is generally called a high-cut filter, or also known as treble cut filter when used for audio applications. A low-pass filter is the contrary of a high-pass filter. A band-pass filter is a merger of a low-pass and a highpass.

\subsection{Wireless Transmission/ Reception}

Wireless module based on IEEE 802.15.4 is used for transmission and receiving the signal. ECG front end circuit is connected with wireless module as remote base station. Receiver module is interfaced with PC at Base station.

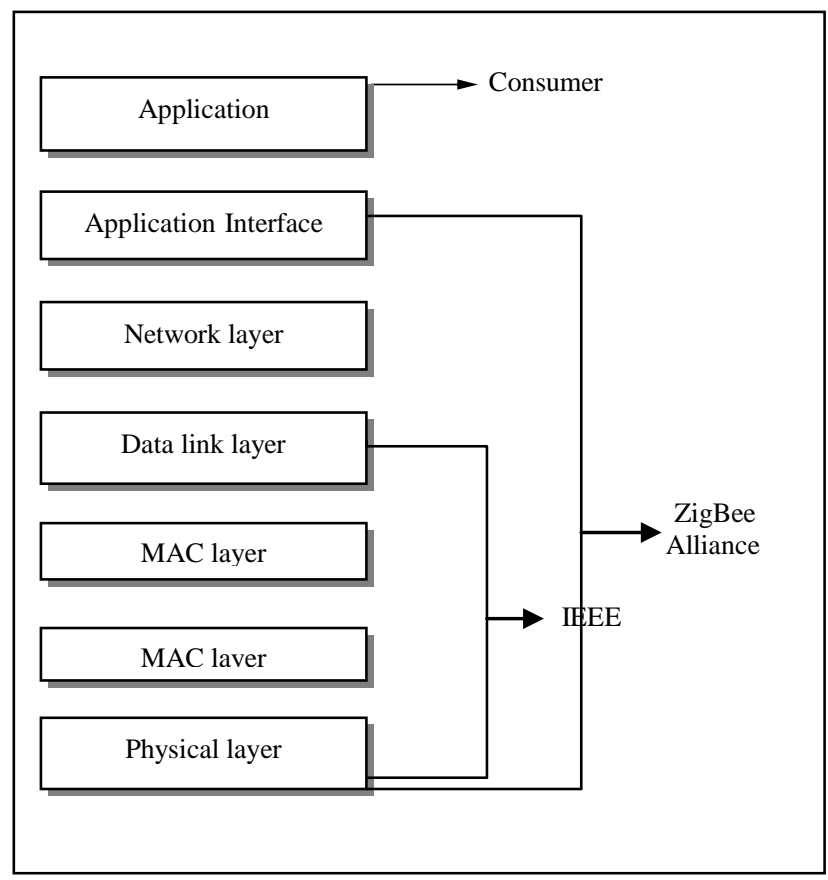

Fig.3 ZigBee Stack

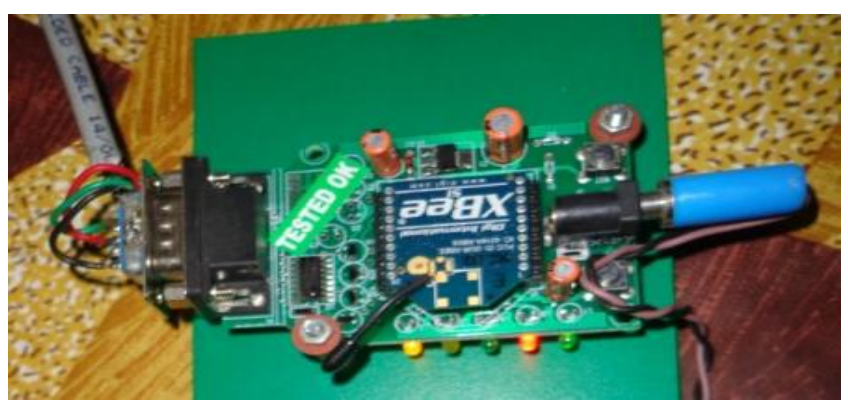

Fig. 4 ZigBee module

ZigBee and ZigBee-PRO Modules are engineered to meet ZigBee/IEEE 802.15.4 standards and also support the exclusive needs of low-cost, low-power wireless sensor networks. Table 1 presents ZigBee Pro technology specifications[15]. 
The modules require minimal power and provide reliable delivery of critical data amongs devices. The modules function within the ISM $2.4 \mathrm{GHz}$ frequency band and are pin compatible with each other.

ZigBee module (Digi International, MN) is chosen as the wireless radio for this study. It is low cost and implements the ZigBee routing protocol over IEEE 802.15.4 standard. ZigBee Stack is shown in figure 3 .

The wireless communication between the portable unit and the access point is established. Since the ZigBee routing protocol is used, the portable device is configured as the end device and the access point is configured as the coordinator. The objective is to evaluate the performance of the wireless network in real time monitoring of ECG using the XBEE radio (Fig. 4). A ZigBee module connected to portable ECG unit forms transmitter and another desktop computer with the second ZigBee module simulates the access point. A physician can access this computer either with remote access or through cloud.

Devices that have a UART interface can connect directly to the pins of the RF module as shown in the Figure 5. Figure shows data flow diagram in UART

The data enters the module UART through the DI pin as an asynchronous serial signal. The signal is idle high when no data is being transmitted. Each data byte consists of a start bit (low), 8 data bits (least significant bit first) and a stop bit (high). The module UART accomplishes jobs, such as time and parity verification, that are required for data communications. Serial communications rely on two UARTs to be configured with compatible settings (baud rate, parity, start bits, stop bits).

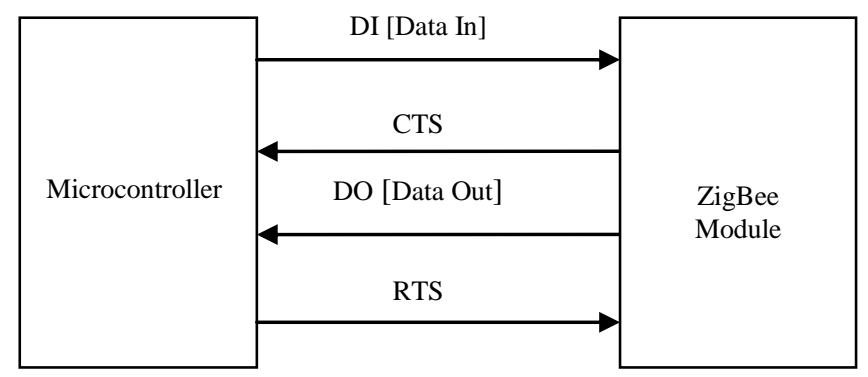

Fig. 5 Data flow diagram in UART

\section{RESULTS}

Experiments were performed on the developed ECG system. Figure shows 3 Cropped Snapshot of the client screen ECG waveform generated by developed system. Generated ECG signal is filtered to remove noise from signals. Data is transmitted in form of packets in zigbee module with inbuilt ADC. The data is stored on data servers and is made accessible universally.

Figure 6 presents cropped Snapshot of the client screen ECG view. The experiments were also performed to test the reliability of the cloud based system. It was tested on hosted website. The table Table 2 shows experimentation done for validating the reliability and in turn availability of the system. The tests were conducted in all days of the week on different time at random and at different locations with help of friends and relatives who tested it voluntarily. It was found that the data was available. It was also extended on fortnightly basis and was found to work efficiently.

\section{REAL TIME ECG DATA}

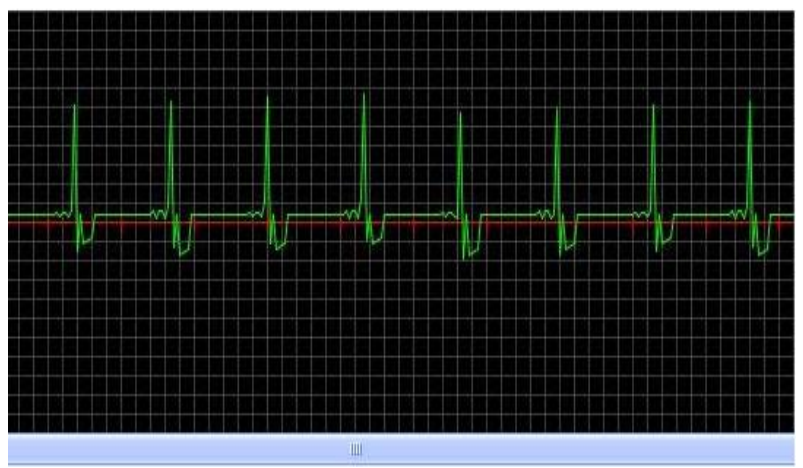

Fig. 6 Cropped Snapshot of the client screen

Table 2. Results of the experimentation done to check availability of the system

\begin{tabular}{|c|c|l|c|}
\hline Sr. No. & $\begin{array}{c}\text { Day of } \\
\text { the week }\end{array}$ & Time & Availability \\
\hline 1 & 1 & $10.00 \mathrm{AM}$ & Yes \\
\hline 2 & 2 & $2.00 \mathrm{PM}$ & Yes \\
\hline 3 & 3 & $7.00 \mathrm{AM}$ & Yes \\
\hline 4 & 4 & $3.45 \mathrm{AM}$ & Yes \\
\hline 5 & 5 & $4.55 \mathrm{PM}$ & Yes \\
\hline 6 & 6 & $11.55 \mathrm{AM}$ & Yes \\
\hline 7 & 7 & $1.55 \mathrm{PM}$ & Yes \\
\hline
\end{tabular}

\section{CONCLUSION}

With aim to find some new solutions for wireless biomedical applications, an ECG acquisition, wireless transmission system and then cloud services makes the system an innovative system. With use of ZigBee malicious users are prohibited from unauthorized access to the data; the power requirements are very low. The system will help to save the life of the cardiovascular patients. It can be used as multipurpose system or can be used in multiple ways. A heart patient can keep this portable device with him and if he feels that, he is having some problem; he can call his doctor and send ECG signals from his home or workplace. The doctor can suggest appropriate action. A physician can seek help of experts from abroad. Similarly, this device can be mounted in ambulance or cardiac van. For continuous monitoring of ECG data it is stored on cloud data servers. As the system is interfaced with cloud to provide reliability of service at relatively low cost. The proposed system is cost effective; it provides availability and reliability and fault tolerant. Thus, significantly enhancing service quality of emergency medical system. 


\section{REFERENCES}

[1] Wei Lin, "Real Time Monitoring of Electrocardiogram Through IEEE802.15.4 Network", Eighth International Conference \& Expo on Emerging Technologies for a Smarter World (CEWIT), February, 2011 , pp. $1-6$.

[2] Maulin Patel and J. Wang, "Applications, Challenges and Prospective In Emerging Body Area Networking Technologies IEEE Wireless Communications, (Volume:17 , Issue: 1 ) IEEE Communications Society, February 2010 pp. $80-88$.

[3] Reza S. Dilmaghani, Hossein Bobarshad, "Wireless Sensor Networks For Monitoring Physiological Signals of Multiple Patients", IEEE Transactions on Biomedical Circuits and Systems, Volume:5 , Issue: 4 August. 2011 pp. 347 - 356.

[4] R. Sukanesh, S. Palanivel Rajan, S. Vijayprasath, S Janardhana Prabhu, P. Subathra, "GSM-Based ECG Tele-Aler T System", International Journal of Computer Science and Application Issue 2010

[5] M. Krishnamurthy And H.M. Rajashekara, "Current Trends In Wireless Technologies In Academic Libraries", DESIDOC Journal Of Library \& Information Technology, Vol. 31, No. 1, pp. 41-48, January 2011.

[6] Carlos Oberdan Rolim, Fernando,Luiz Koch, Carlos Becker Westphall, Jorge Werner, Armando Fracalossi and Giovanni Schmitt Salvador, "A Cloud Computing Solution For Patient's Data Collection In Health Care Institutions", Second International Conference on ehealth, Telemedicine, and Social Medicine,Oct. 2010.

[7] Sasan Adibi, "Link Technologies and Blackberry Mobile Health (Mhealth) Solutions: A Review", IEEE Transactions on Information Technology in Biomedicine, Volume: 16, Issue: 4 , July 2012,pp. 586-597.
[8] Hak Jong Lee, Sun-Hee Lee, Kyoo Seob Ha, Hak Chul Jang, Woo Young Chung, Ju-Young Kim, Yoon Seok Chang, Dong Hyun Yoo. "Ubiquitous healthcare service using Zigbee and mobile phone for elderly patients", I. J. Medical Informatics, 2009, 78(3):193198.

[9] Piotr Augustyniak, "Autoadaptivity and Optimization", Distributed ECG Interpretation", IEEE Transactions on Information Technology In Biomedicine, Vol. 14, NO. 2, March 2010.

[10] Xiuxia Yu, Kebingwu, "Design and Implementation Of ECG Wireless Transmission System Based On ARM9", International Conference On Computer, Mechatronics, Control and Electronic Engineering (CMCE),June 2010.

[11] George Qi Gao, "Computerised Detection \& Classification of Five Cardiac Conditions", Master of Engineering Thesis submitted to Auckland University New Zealand May 2003.

[12] Mariana Carroll, Paula Kotzé, Alta van der Merwe. "Securing Virtual and Cloud Environments". In: Cloud Computing and Services Science, Service Science: Research and Innovations in the Service Economy), edited by I. Ivanov et al., Springer Science and Business Media, 2012.

[13] Kyungtae Kang, Kyung-Joon Park, Ae-Jin Song, Chang-Hwan Yoon, And Lui Sha, "A Medical-Grade Wireless Architecture For Remote Electrocardiography" IEEE Transactions on Information Technology In Biomedicine, Vol. 15, No. 2, March 2011.

[14] S. Khoor, J. Nieberl, K. Fugedi, E Kail, "Telemedicine ECG- Telemetry With Bluetooth Technology", Computers In Cardiology 585-588, January 2001

[15] Patrick Kinn "ZigBee Technology: Wireless Control that Simply Works", ZigBee white Paper, ZigBee Allice 2003 